\title{
RETOS DE LAS INSTITUCIONES DE EDUCACIÓN SUPERIOR FRENTE A LA SITUACIÓN ACTUAL DEL DEPARTAMENTO DE BOYACÁ RESPECTO A LOS INDICADORES DEL ÍNDICE DEPARTAMENTAL DE INNOVACIÓN
}

\section{CHALLENGES OF THE INSTITUTIONS OF HIGHER EDUCATION AGAINST THE CURRENT SITUATION OF THE DEPARTMENT OF BOYACÁ REGARDING THE INDICATORS OF THE INNOVATION DEPARTMENTAL INDEX}

\section{Liseth Johana Becerra Duitama1.}

Erika Sofía Olaya Escobar².

\section{Hugo Fernando Castro Silva ${ }^{3}$}

Universidad Pedagógica y Tecnológica de Colombia

$1 \quad$ Universidad Pedagógica y Tecnológica de Colombia, https://orcid.org/0000-0002-2630-1157. Candidata a Magíster en Administración. Especialista en Alta Gerencia de Empresas. Administradora Turística y Hotelera. Estudiante Investigadora.

2 Escuela Colombiana de Ingeniería Julio Garavito, https://orcid.org/0000-0001-6254-1169 y https://scholar. google.com/citations? $h /=$ es\&user $=G 36 x L 9 w A A A A J \& v i$ ew_op=list_works. Profesora Asociada. Ph.D en Dirección y Administración de Empresas. M.Sc En Ingeniería: Materiales y Procesos. Ingeniero Industrial.

3 Universidad Pedagógica y Tecnológica de Colombia, https://orcid.org/0000-0001-6020-402X.m Profesor de Planta. Ph.D en gerencia de proyectos. MSc En ingeniería industrial. Ingeniero Industrial.

\section{RESUMEN}

Colombia, consciente de la importancia de la innovación como un elemento fundamental en la generación de valor y el crecimiento económico ha venido incorporando en su agenda competitiva actividades de Ciencia, Tecnología e Innovación; sin embargo, su impacto aún es limitado en el desarrollo del país. Al revisar el panorama nacional, en Boyacá falta 
camino por recorrer para capitalizar la economía del conocimiento y consolidar una oferta productiva más sofisticada que propenda por un departamento más innovador y competitivo. Al tener en cuenta lo anterior, es evidente la ausencia de estudios que permitan identificar la situación actual del departamento frente a indicadores de innovación como un insumo para investigaciones futuras. El desarrollo de este trabajo permite determinar la situación actual del Departamento frente a los indicadores del Índice departamental de innovación a través del desarrollo de cinco fases. En primer lugar, se hizo una indagación para determinar cómo se encuentra Colombia frente a estándares internacionales de innovación mediante el análisis del índice Global de Innovación. En segundo lugar, se analizó la información recolectada para determinar las brechas del departamento de Boyacá frente a indicadores del Índice Departamental de Innovación 2018. En tercer lugar, se identificaron y analizaron los indicadores del Índice Departamental de Innovación en los cuales las Instituciones de Educación Superior tienen mayor incidencia para su aporte. En cuarto lugar, se analizaron los resultados. Finalmente se plantearon conclusiones y recomendaciones.

\section{PALABRAS CLAVE}

Índice Global de Innovación, Índice departamental de innovación, insumos de innovación, resultado de innovación, razón de eficiencia, Instituciones de Educación Superior

\section{ABSTRACT}

Colombia, aware of the importance of innovation as a basic element in value generation and economic growth has been incorporating Science, Technology and Innovation activities in its competitive agenda, however, its impact is still limited in the development of the country .Reviewing the national scene, in Boyacá, there is still a long way down to capitalize on the knowledge economy and consolidate a more sophisticated productive offer provided by a more innovative and competitive department. In view of the above, it is evident the absence of studies to identify the current situation of the department against innovation indicators as an input for future research. The development of this work allows us to identify the current situation of the department against the indicators of the Departmental Innovation Index through the development of five phases. First, an inquiry was made to determine how Colombia is facing international innovation standards through the analysis of the Global Innovation Index. Second, the information collected was analyzed to determine the gaps of the department of Boyacá against indicators of the Departmental Index of Innovation 2018. Third, the indicators of the Departmental Index of Innovation in which Higher Education Institutions have greater incidence for their contribution were identified. Fourth, the results were analyzed. Finally, conclusions and recommendations were raised.

\section{KEYWORDS}

Global Innovation Index, Departmental Innovation Index, innovation inputs, result of innovation, efficiency ratio, Higher Education Institutions

\section{INTRODUCCIÓN}

El fomento de estudios mundiales sobre innovación, la orientación de políticas y el surgimiento de mejores prácticas, ha reflejado durante años la necesidad de disponer de un sistema de medición a través del cual evaluar la innovación. Por tal motivo, desde el 2007, la Organización Mundial de la Propiedad Intelectual (OMPI), la Universidad de Cornell y la Escuela de Negocios INSEAD elaboraron de forma conjunta, el Índice Global de Innovación (GII), como un indicador que permite determinar las capacidades y los resultados en el ámbito de innovación de las economías del mundo. 
El índice está compuesto por dos subíndices insumos y resultados. Su relación se denomina razón de eficiencia, la cual expresa qué tan eficienteesun país en lageneración de innovación teniendo en cuenta la calidad y cantidad de los insumos empleados para obtener resultados (Dutta, Lanvin, \& Wunsch-Vincent, 2018). Para 2018, el tema del GII fue "Energizando el mundo con la innovación" y reflejó las capacidades de innovación y los resultados de 126 países, a través de 80 indicadores, agrupados en 21 subpilares, siete pilares y dos subíndices. Como se observa en la Figura 1 el subíndice de entrada o insumos se compone de cinco pilares: instituciones, capital humano e investigación, infraestructura, sofisticación del mercado y sofisticación de los negocios. El subíndice de salida o resultados se compone de dos pilares: producción de conocimientos y tecnología, y producción creativa.

Cada año el Índice clasifica los países en función de sus resultados; de esta manera, presenta los datos a nivel mundial resaltando las 20 primeras economías líderes en innovación. Adicionalmente clasifica los países en siete regiones: 1. Norte América, 2. Europa, 3. África del Norte y Asia Occidental, 4. Asia Sudoriental, Asia oriental y Oceanía, 5. Latinoamérica y el Caribe, 6. África Subsahariana y Asia Central y del Sur. Finalmente los clasifica por ingresos, al establecer cuatro grupos de países: 1. ingresos altos, 2. ingresos medianos - altos, 3. ingresos medianos - bajos y 4 . Ingresos bajos.

Figura 1. Conformación del Índice Global de Innovación 2018

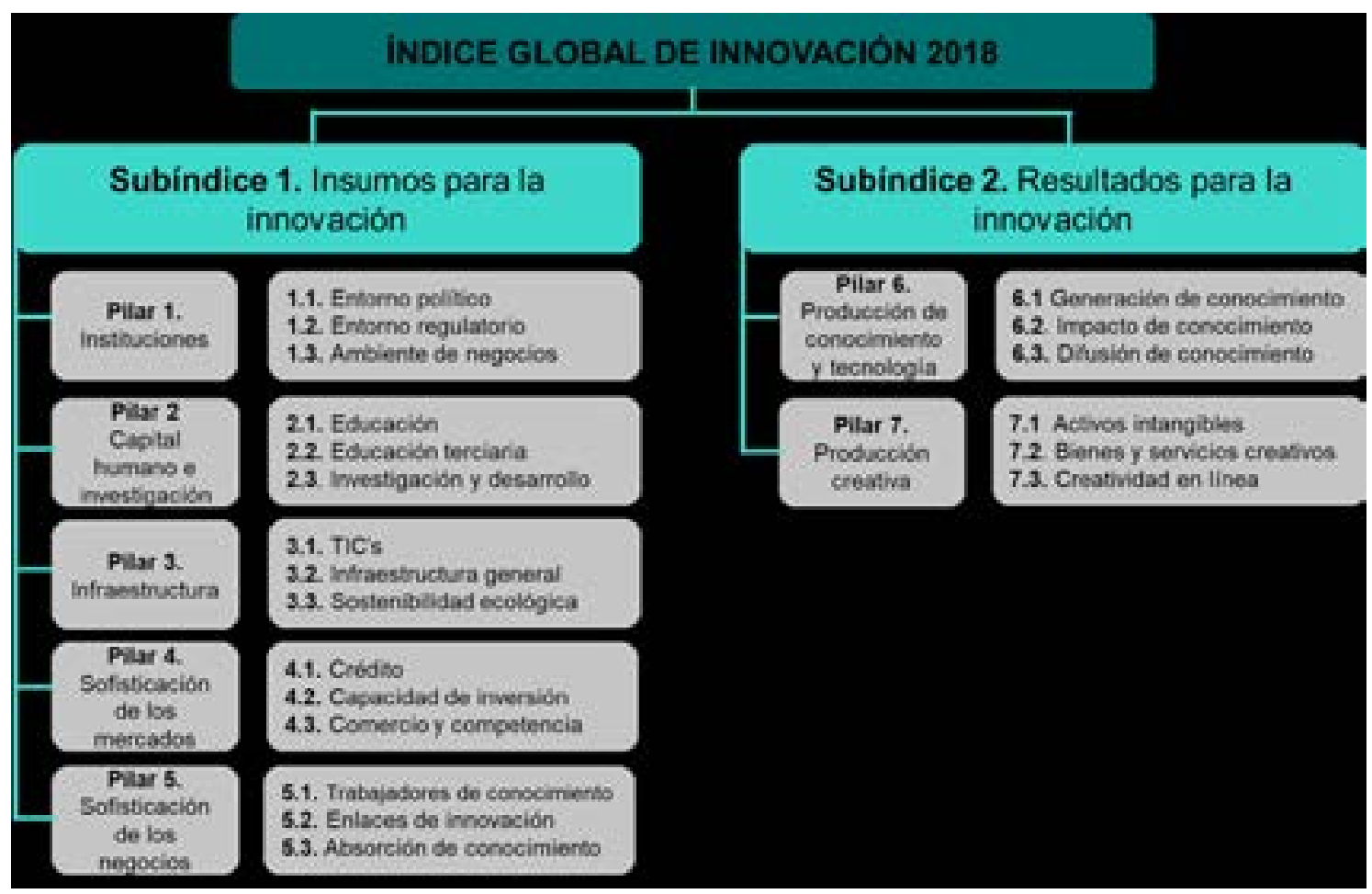

Fuente: elaboración propia a partir del Índice Global de Innovación, 2018

En 2018, y por octavo año consecutivo, Suiza encabeza la clasificación, en la cual de 47 economías de ingresos altos 19 se ubican dentro de los 20 puestos principales, siendo
China una excepción, al ocupar el puesto 17. Cabe resaltar que, en 2016 este país pasó a ser la primera economía de ingresos medianos - altos que ocupa un puesto principal al obtener 
muy buenas calificaciones en los diferentes pilares resultados (Dutta et al., 2018).

\section{COLOMBIA FRENTE A LOS INDICADORES DEL ÍNDICE GLOBAL DE INNOVACIÓN}

EI GII define la eficiencia de los esfuerzos locales con relación a los resultados es decir la relación de los resultados dividido entre el valor del subíndice insumos. Si esta razón de eficiencia es cercana o mayor a uno, indica que el país tiene un ecosistema de innovación robusto que transforma adecuadamente insumos en productos. Por contrario, si la razón de eficiencia es próxima a cero, el país enfrenta dificultades que le impiden convertir capacidades e insumos en resultados efectivos de conocimiento e innovación. En América Latina se destacan Costa Rica y Uruguay quienes lideran en términos de eficiencia en la región con razones de 0,68 y 0,64, mientras que Colombia y Perú son los más ineficientes en Sudamérica con un 0.5 (DNP, 2018)

Colombia que hace parte del grupo de economías de ingresos medianos - altos, en el 2018 mejoró dos posiciones al pasar del lugar 65 al 63. Sin embargo, se observa una disminución en el puntaje al pasar de 34,8 a 33,8, generado por la variación negativa de los subíndices insumos $(-0,7)$ y de resultados $(-1,2)$. En el subíndice insumos, Colombia obtuvo menores puntajes en 4 de 5 pilares, destacándose el pilar capital humano e investigación como su mayor debilidad y respecto al subíndice de resultados se identifica el pilar producción creativa como la segunda debilidad en el sistema de innovación colombiano. Según el Departamento de Planeación (DNP) en el informe para Colombia del Índice Global de Innovación 2018 esa obtención "aumenta la presión sobre el sistema de innovación nacional que continúa con dificultades para convertir en resultados tangibles las inversiones efectuadas en construcción de capacidades y recursos" (DNP \& OCyT, 2018).

Alineados con el esfuerzo mundial de medir la innovación y con el fin de cumplir los lineamientos planteados por la Organización para la Cooperación y el Desarrollo Económico (OCDE) en el Manual de Oslo sobre este tema, en el contexto nacional, desde el año 2016 el Departamento Nacional de Planeación y el Observatorio Colombiano de Ciencia y Tecnología (OCyT), han elaborado el Índice Departamental de Innovación para Colombia (IDIC) con el fin de identificar las diferencias a nivel territorial en términos de innovación a través de una herramienta que permite hacer seguimiento y análisis al desarrollo de las capacidades de generación y apropiación de conocimiento e innovación de los departamentos de Colombia (DNP, OCyT \& Consultores, 2015).

EI IDIC se basa en la metodología utilizada por el GIl y en los parámetros planteados por la OCDE (2008) en su manual para la elaboración de indicadores compuestos. Estos indicadores, se construyen a partir de las versiones del GIl de los años 2014, 2016 y 2018, y son seleccionados a partir de su importancia, los soportes teóricos generados por la revisión de literatura, consulta a expertos, pertinencia departamental, cubrimiento y alcance geográfico y, la facilidad, accesibilidad y actualización a lo largo del tiempo. En algunos casos se utilizaron indicadores alternativos a los planteados en el GIl cuando no fue posible encontrar la información precisa en las diferentes regiones del país, sin alterar de manera considerable su sentido y aporte al Índice.

En este sentido, el IDIC clasifica los departamentos por nivel de desempeño en cinco grupos: alto, medio-alto, medio, mediobajo y bajo. En el Figura 2 , se puede observar que, en 2018, se evaluaron 31 departamentos siendo Cundinamarca (Incluido Bogotá) y 
Antioquía quienes encabezan el escalafón del IDIC presentando un nivel de desempeño alto, seguidos de Santander, Atlántico, Valle del Cauca, Risaralda y Caldas, quienes presentan un nivel medio-alto de desempeño. En el nivel de desempeño medio se ubican Quindío, Bolívar, Cauca, San Andrés, Boyacá, Tolima, Nariño, Meta, Huila y Norte de Santander, seguidos

Figura 2. Ranking del Índice Departamental de Innovación para Colombia, 2018

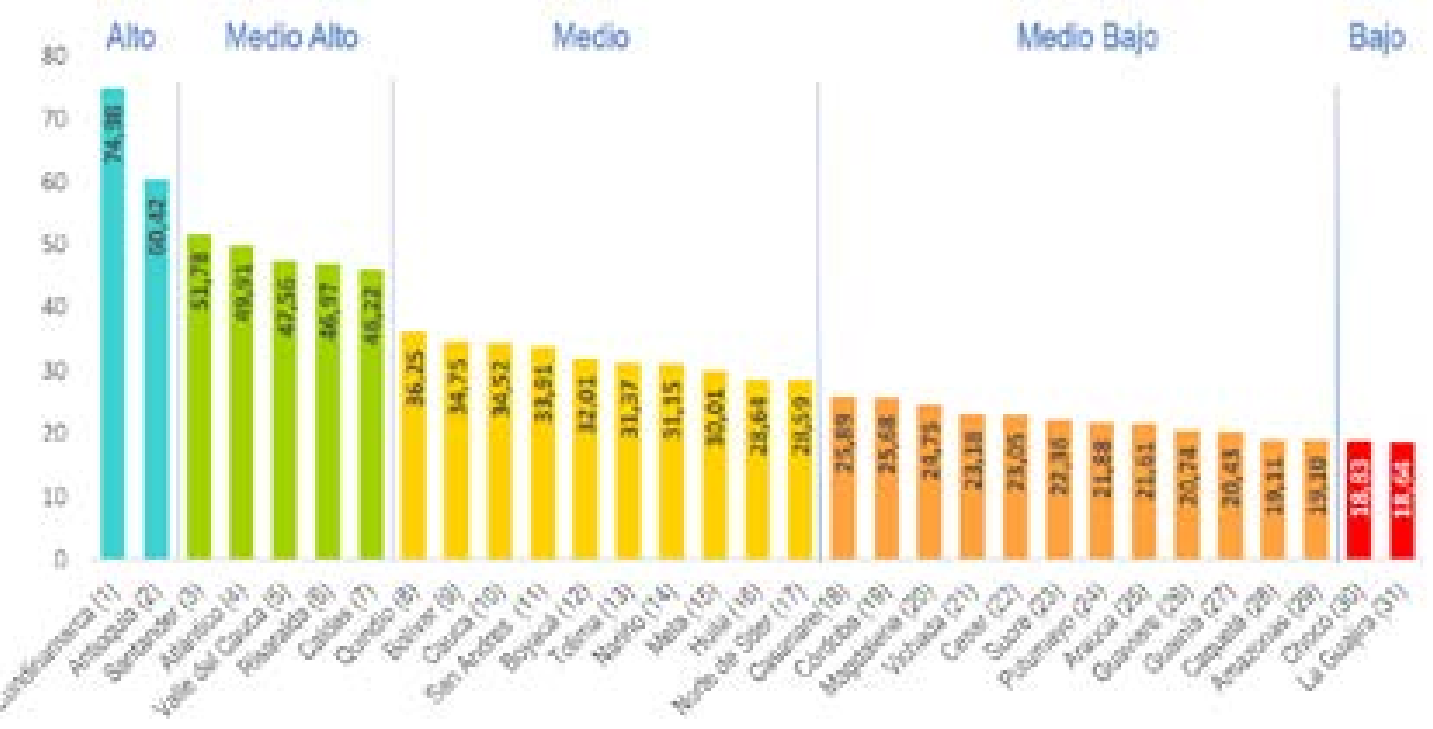

de Casanare, Córdoba, Magdalena, Vichada, Cesar, Sucre, Putumayo, Arauca, Guaviare, Guanía, Caquetá y Amazonas, quienes muestra un nivel medio-bajo de desempeño. Por su parte, la Guajira y Chocó fueron los únicos departamentos que se ubican en un nivel de desempeño bajo (DNP \& OCyT, 2018)

Fuente: DNP \& OCyT. (2018)

En el GIl 2018 los pilares con el mejor desempeño de Colombia están en el subíndice de insumos: sofisticación de mercados (40), infraestructura (40) y sofisticación de negocios (56). A pesar de la buena ubicación del pilar de sofisticación de mercados, en 2018 Colombia cedió nueve posiciones en este pilar. Por otra parte, los pilares más rezagados en el país son producción creativa (77) y capital humano e investigación (78). En este último se destaca el retroceso de 13 posiciones en el subpilar investigación y desarrollo (DNP, 2018)

\section{INDICE DEPARTAMENTAL DE INNOVACION DEPARTAMENTO DE BOYACA}

Los resultados del informe del IDIC 2018 revelan que el departamento de Boyacá tiene desempeños altos en el subíndice de insumos, pero muy bajos en el subíndice de resultados. Generando una razón de eficiencia de 0.42 , por lo tanto, se considera una combinación poco eficiente para la generación de innovación teniendo en cuenta la calidad y cantidad de los insumos empleados para obtener resultados robustos. Este hecho ha generado un interés académico para abordar este trabajo, puesto que a pesar de tener las condiciones de entrada necesarias para tener un ecosistema de innovación robusto en el Departamento los resultados aún no se hacen evidentes.

En la Figura 3, se muestra que en el subíndice de insumos, se resalta el pilar de capital humano e investigación el cual presentó 
su mejor desempeño con un puntaje de 55,13 y séptimo lugar entre todos los departamentos del estudio; producto del buen comportamiento de los subpilares de educación secundaria y media el cual ocupo el tercer lugar, subpilar de educación superior e investigación y desarrollo que cada uno ocupo el noveno lugar. Por su parte, el subpilar con desempeño más bajo fue el de capacidad de inversión, el cual ocupo del puesto 17 y se evidencia como crítico para el departamento. Respecto al subíndice de resultados presenta debilidades importantes, como lo refleja el pilar de producción de conocimiento y tecnología, en el que este territorio se clasificó en la posición 20, este resultado fue afectado directamente por el subpilar de impacto del conocimiento donde se ocupó la posición 23 , difusión de conocimiento en el puesto 18 y creación de conocimiento en el puesto 14.

Figura 3. Desempeño por pilares y subpilares para el Departamento de Boyacá.

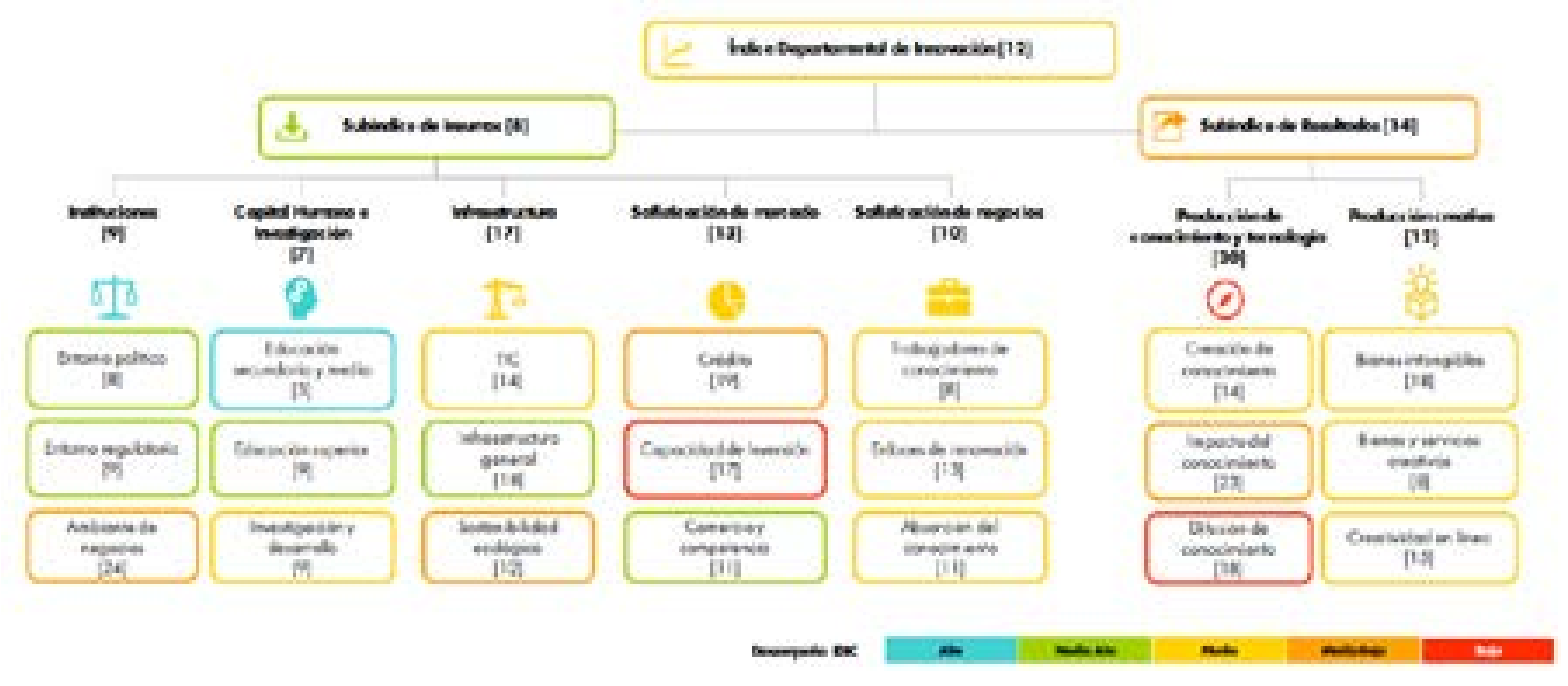

Fuente: DNP \& OCyT. (2018)

En este contexto, se puede decir que Boyacá en el entorno colombiano es un departamento que presenta debilidades en sus capacidades para la innovación puesto que la razón de eficiencia está muy por debajo de 1 . Por ende, es necesaria la articulación entre la academia, gobierno y sector empresarial para lograr una mayor articulación del Sistema Nacional de Ciencia, Tecnología e Innovación que fortalezcan la eficiencia y capacidad de innovación.

\section{RETOS DE LAS INSTITUCIONES DE EDUCACIÒN SUPERIOR DEL DEPARTAMENTO DE BOYACÁ FRENTE A LOS INSUMOS PARA LA INNOVACIÓN}

Como se presenta en la Figura 1, el subíndice de Insumos para la innovación se compone de cinco pilares: instituciones, capital humano e investigación, infraestructura, sofisticación del mercado, y sofisticación de negocios. Sin embargo, y dado que la unidad de análisis de este trabajo son las Instituciones de Educación Superior (IES), se analizaron los indicadores relacionados con docencia, investigación y transferencia (Etzkowitz \& Leydesdorff, 2000; Gunasekara, 2006). En la 
Figura 4 se identifican los subíndices, pilares, subpilares e indicadores afines a las funciones misionales de estas instituciones y por lo tanto su desempeño puede relacionarse con el esfuerzo que se haga desde la academia para su fortalecimiento. Teniendo en cuenta que este estudio se centra en el departamento de Boyacá se identificaron los indicadores con mayor fortaleza para este departamento y que a su vez se relacionan con las funciones misionales de las IES

Figura 4. Indicadores relacionados con las Instituciones de Educación Superior

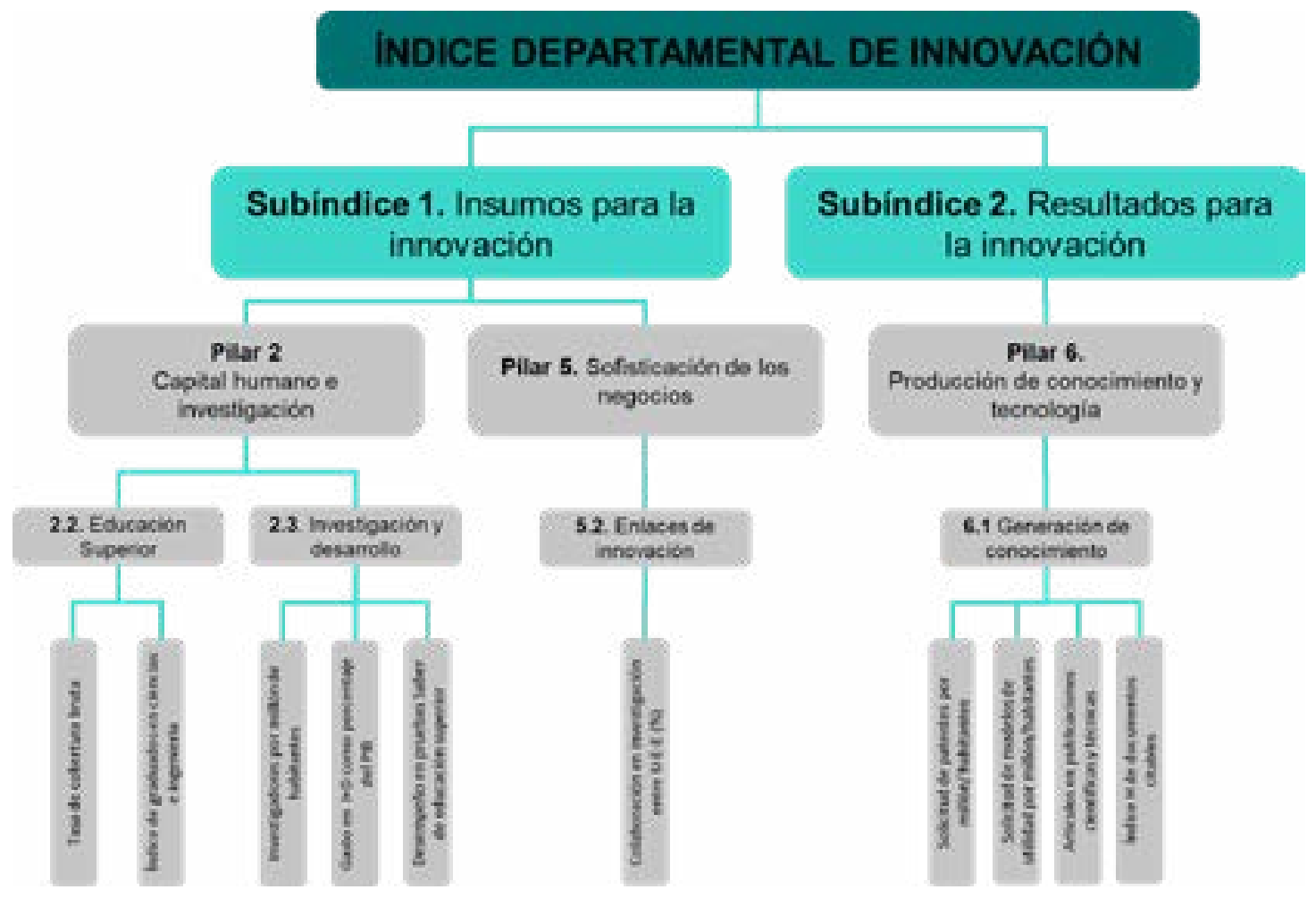

Fuente: elaboración propia a partir del Índice

Global de Innovación, 2018

De los indicadores seleccionados, los catalogados en el IDIC con alto desempeño se pueden resaltar: la tasa de cobertura bruta en educación superior, artículos en publicaciones científicas y técnicas, por su parte con desempeño medio-alto se identificó el de investigadores por cada millón de habitantes.
Por otra parte, los indicadores que presentan mayores debilidades con un desempeño medio-bajo son: gasto en investigación y desarrollo como porcentaje del PIB, desempeño en pruebas Saber de educación terciaria, colaboración en investigación entre empresas y otras organizaciones de conocimiento (\%) y 
solicitudes de modelos de utilidad por millón de habitantes. Es importante resaltar que de los indicadores seleccionados para este trabajo no se reporta ninguno con desempeño bajo (DNP \& OCyT, 2018)

\section{PILAR 2. CAPITAL HUMANO E INVESTIGACIÓN}

Como se observa en la Figura 3 en el subíndice de insumos para la innovación del departamento de Boyacá, se pueden resaltar los subpilares de educación superior e investigación y desarrollo que corresponden al pilar de capital humano e investigación, el cual es el pilar con mejor desempeño para este departamento.

\section{SUBPILAR EDUCACIÓN SUPERIOR}

Este subpilar captura la información sobre la cobertura en educación superior; la prioridad asignada a áreas del conocimiento que son esenciales para la innovación (ciencias e ingenierías) y la movilidad de estudiantes extranjeros que juega un papel clave en el intercambio de ideas y habilidades necesarias para innovar (DNP, OCyT, \& Consultores, 2015). Como se evidencia en la Figura 4 , este subpilar corresponde al pilar de capital humano e investigación que a su vez corresponde al

Figura 5. Evolución de las matriculas en las IES en el Departamento de Boyacá subíndice de insumos para la innovación.

El desempeño del departamento de Boyacá respecto a este subpilar evidencia que mantiene un índice superior a la media nacional. Según el Sistema Nacional de Información de la Educación Superior (SNIES) en su Informe Departamental de Educación Superior para Boyacá del 2017 (https://bit.ly/31HNM3L), la tasa de cobertura bruta en educación superior para el departamento es de $56,3 \%$ mientras a nivel nacional es del $52,8 \%$. El número total de matrículas en educación superior para el 2017 para Boyacá correspondiente a 69.413 respecto 2.446.314 en todo el país. En la Figura 5 se presenta la tendencia creciente de las matriculas en educación superior en el departamento en el periodo comprendido entre el 2010 al 2017, evidenciándose la apuesta del departamento para el fortalecimiento de educación superior. A pesar de que las IES oficiales siguen teniendo la mayor proporción de matrículas con un crecimiento sostenido, se reconoce el incremento de las matriculas de más del doble para las IES privadas en el mismo periodo de tiempo. Ahora bien, respecto a las matriculas por sector para el 2017 el $70.06 \%$ corresponde a matriculas en el sector oficial y $29.94 \%$ en el sector privado.

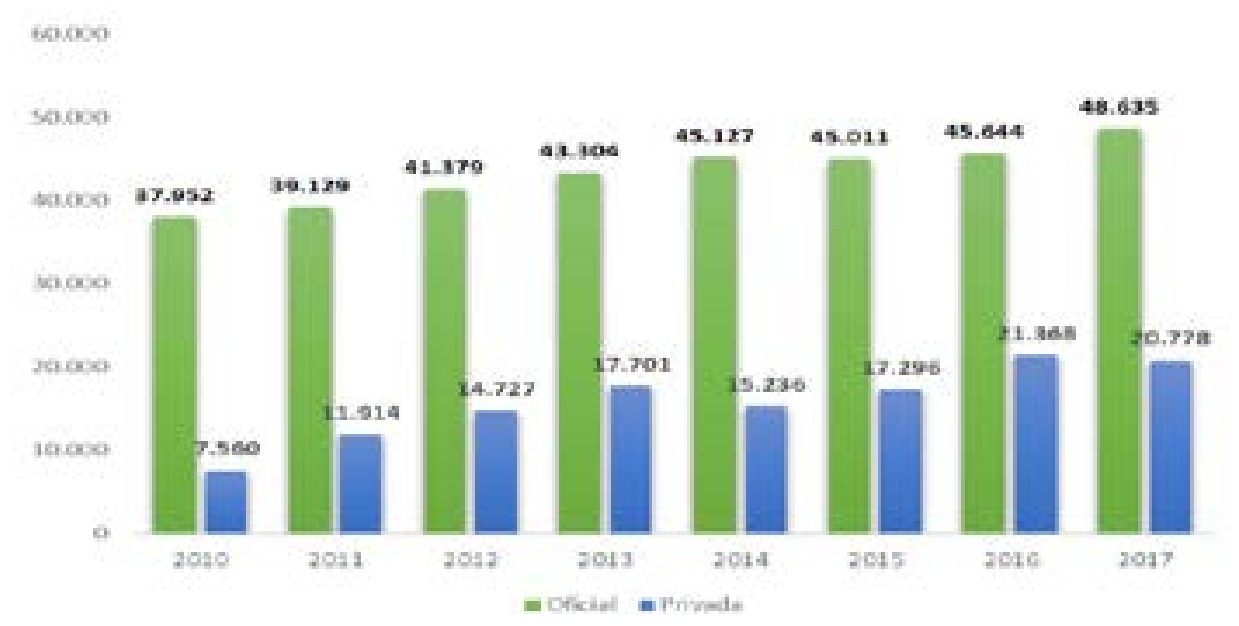

Fuente: SNIES (2017) 
Figura 6 se presenta la proporción de matrículas correspondientes a programas de posgrado con un $11 \%$ y respecto al pregrado un $89 \%$ en el 2017. Este indicador es muy importante para evidenciar el fortalecimiento de la región en personal altamente capacitado necesario para soportar las actividades de Ciencia, Tecnología e Innovación e iniciar a apalancar una economía basada en conocimiento para la región, teniendo en cuenta que la proporción a nivel nacional es de $93 \%$ para pregrado versus el $7 \%$ para posgrados en el mismo periodo de tiempo.

Respecto a las matriculas por nivel de formación en la Figura 7 se presenta la tendencia desde el 2010 hasta el 2017, evidenciando que el nivel universitario es el más relevante para la región no solo por el volumen sino porque ha tenido un crecimiento sostenido en el periodo de tiempo evaluado. Al cotejarlo con la Figura

Figura 7. Graduados por nivel de formación en el Departamento de Boyacá 2010-2016
Figura 6. Matrículas según el nivel de formación para BOYACÁ -2017

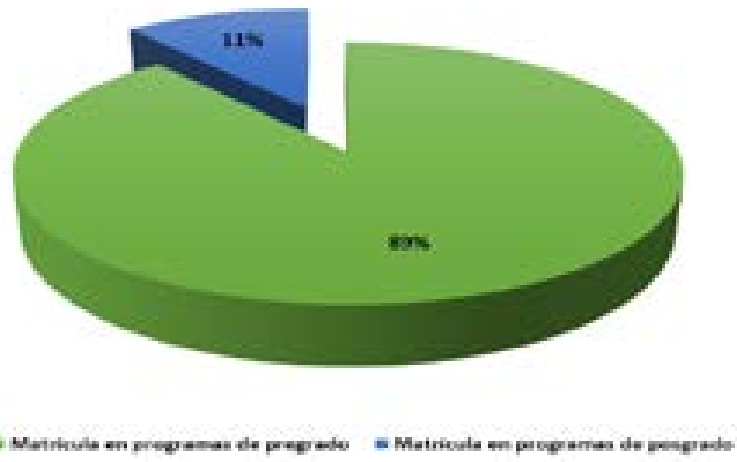

Fuente: SNIES (2017)

6 , se puede analizar que, aunque la formación posgradual aún es baja en proporción con los demás niveles de formación, ha tenido un incremento importante y sostenido. Al revisar los datos del 2017 se corrobora este análisis con un $65.81 \%$ a nivel universitario, $8.68 \%$ a nivel especialización, el $2.08 \%$ a nivel maestría, y el $0.18 \%$ a nivel doctorado.

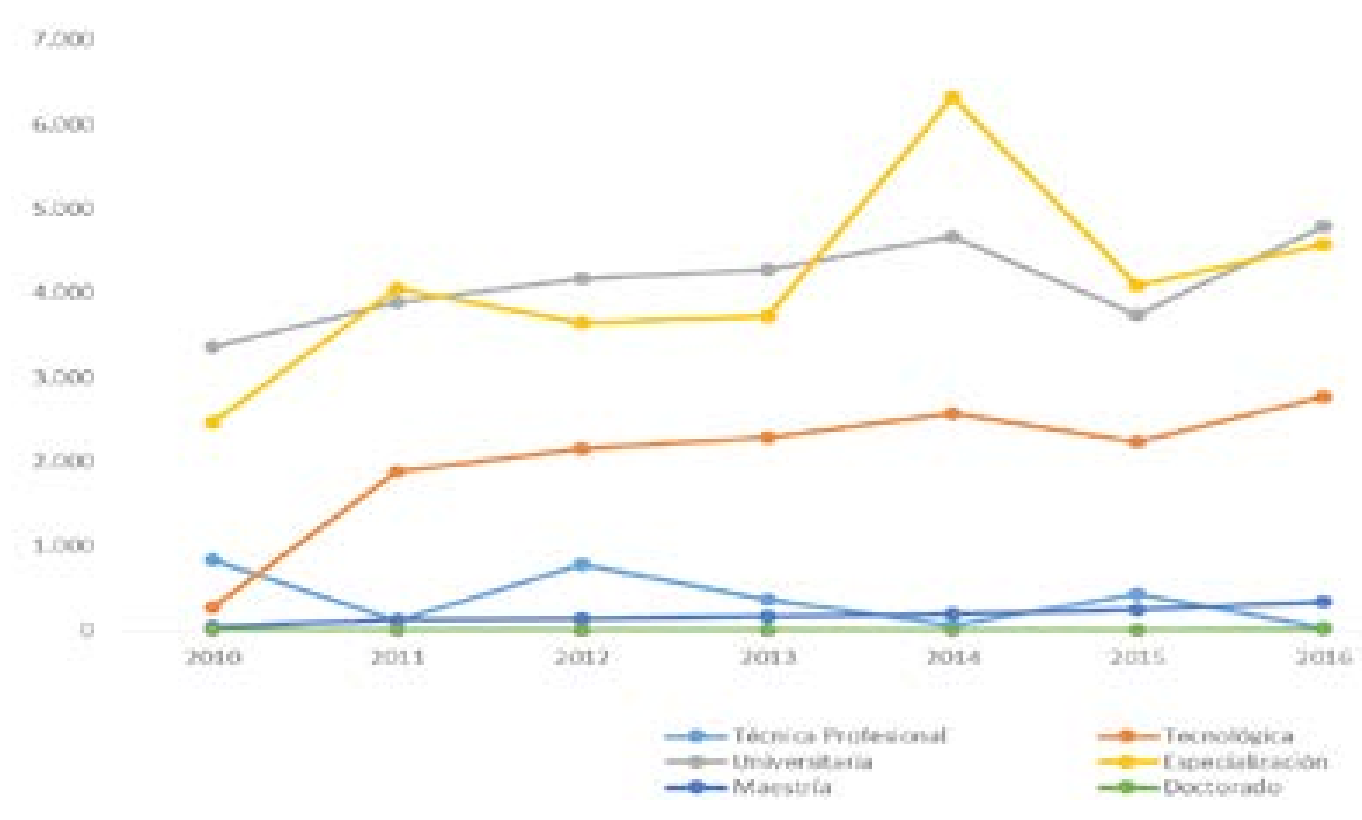

Fuente: SNIES (2017) 
Por otra parte, la Figura 8 muestra la matriculas por área de conocimiento y se evidencia la vocación de la región en áreas como ingeniería, arquitectura y urbanismo con un $31 \%$, en segundo lugar economía y administración con el $26 \%$, seguido de ciencias de la educación con el $19 \%$, en cuarto lugar las ciencias sociales y humanas con $11 \%$. En proporciones menores se encuentran ciencias de la salud con $5.03 \%$, agronomía, veterinaria y afines con el $4 \%$, el $2.97 \%$ de matemáticas y ciencias naturales y finalmente con el $1.53 \%$ bellas artes.

Figura 8. Matriculados por área de conocimiento 2017

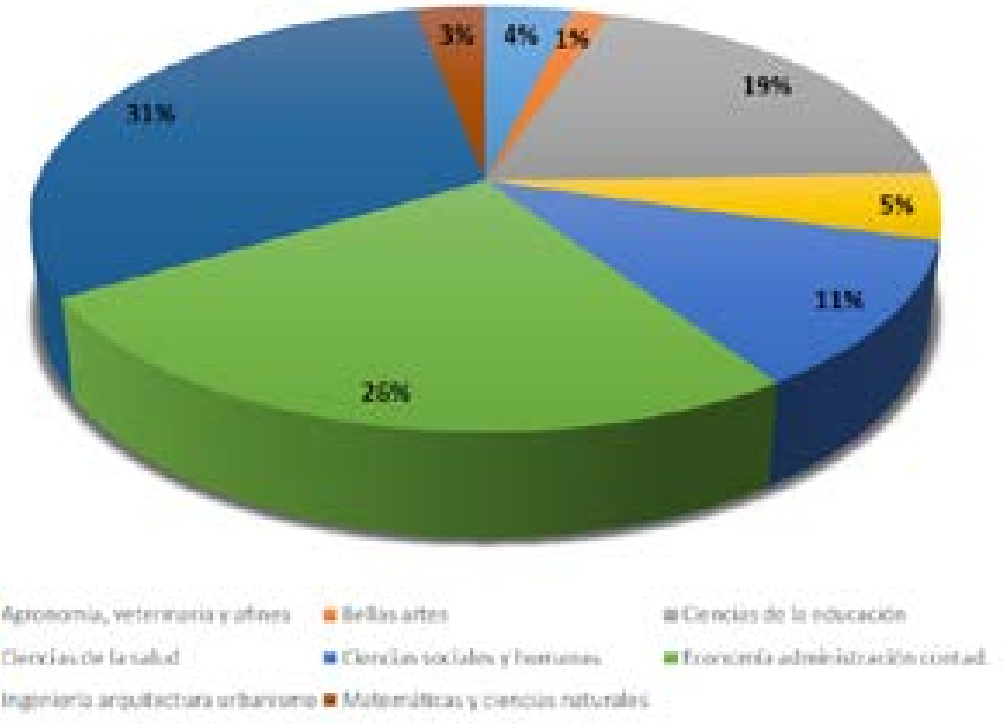

Fuente: SNIES (2017)

De la misma forma en la Figura 9, se observa las potencialidades de la región en $I+D+i$, dado que se evidencia la tendencia creciente en áreas STEM (Ciencias de la salud, ingeniería arquitectura urbanismo, matemáticas y ciencias naturales) desde el 2010 hasta el 2017.

Figura 9. Evolución de las matrículas STEM respeto al total por área de conocimiento

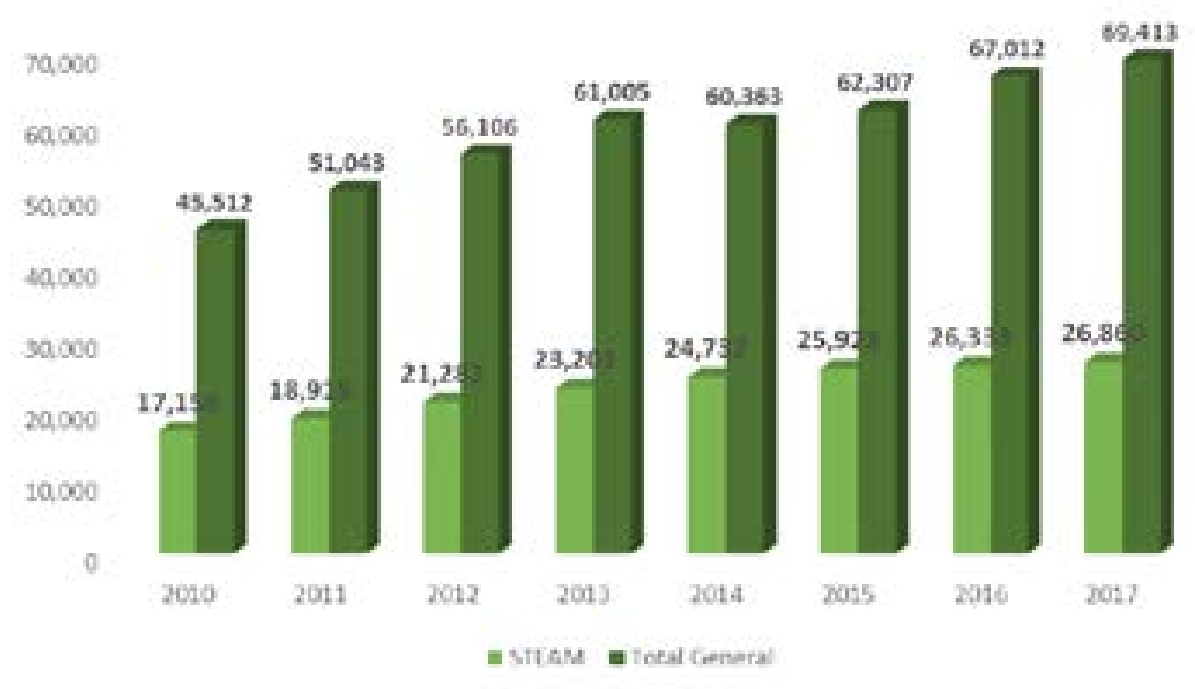

Fuente: SNIES (2017) 


\section{SUBPILAR INVESTIGACIÓN Y DESARROLLO}

Siendo las IES un elemento fundamental para el desarrollo armónico del modelo de Triple Hélice III, requieren de una formación profesional en investigación científica, que ayude a generar procesos de innovación que apalanquen el desarrollo de nuevos conocimientos y enfrentar los retos que el mundo moderno exige (Castillo, 2010). En este sentido, el subpilar investigación y desarrollo mide el nivel y calidad de las actividades de I+D a través de indicadores asociados al número de investigadores, el gasto bruto en $I+D$, Intensidad del gasto empresarial en I+D (\%) y desempeño en pruebas Saber de educación superior (DNP et al., 2015). En este subpilar es importante determinar si las IES presentes en la región cuentan con insumos en innovación reflejados en sus capacidades de

Figura 10. ACTI e I+D como porcentaje del PIB en Boyacá. investigación científica, por tanto, se analizan los indicadores en los cuales las IES aportan directamente, por tal razón se descarta del análisis el indicador intensidad del gasto empresarial en I+D (\%).

Teniendo en cuenta el informe de los Indicadores de Ciencia, Tecnología e Innovación para Boyacá (OCITEB \& UPTC, 2018), el crecimiento de las actividades de Ciencia, Tecnología e Innovación (ACTI) respecto al PIB Departamental, presenta un aumento que se acentúa a partir del año 2012, logrando un porcentaje de $0,25 \%$ en 2016 en donde disminuye un poco para el 2017. En el caso de $I+D$, se puede decir que durante los dos últimos años se mantiene sobre un porcentaje del $0.080 \%$, tal y como puede observarse en la Figura 10.

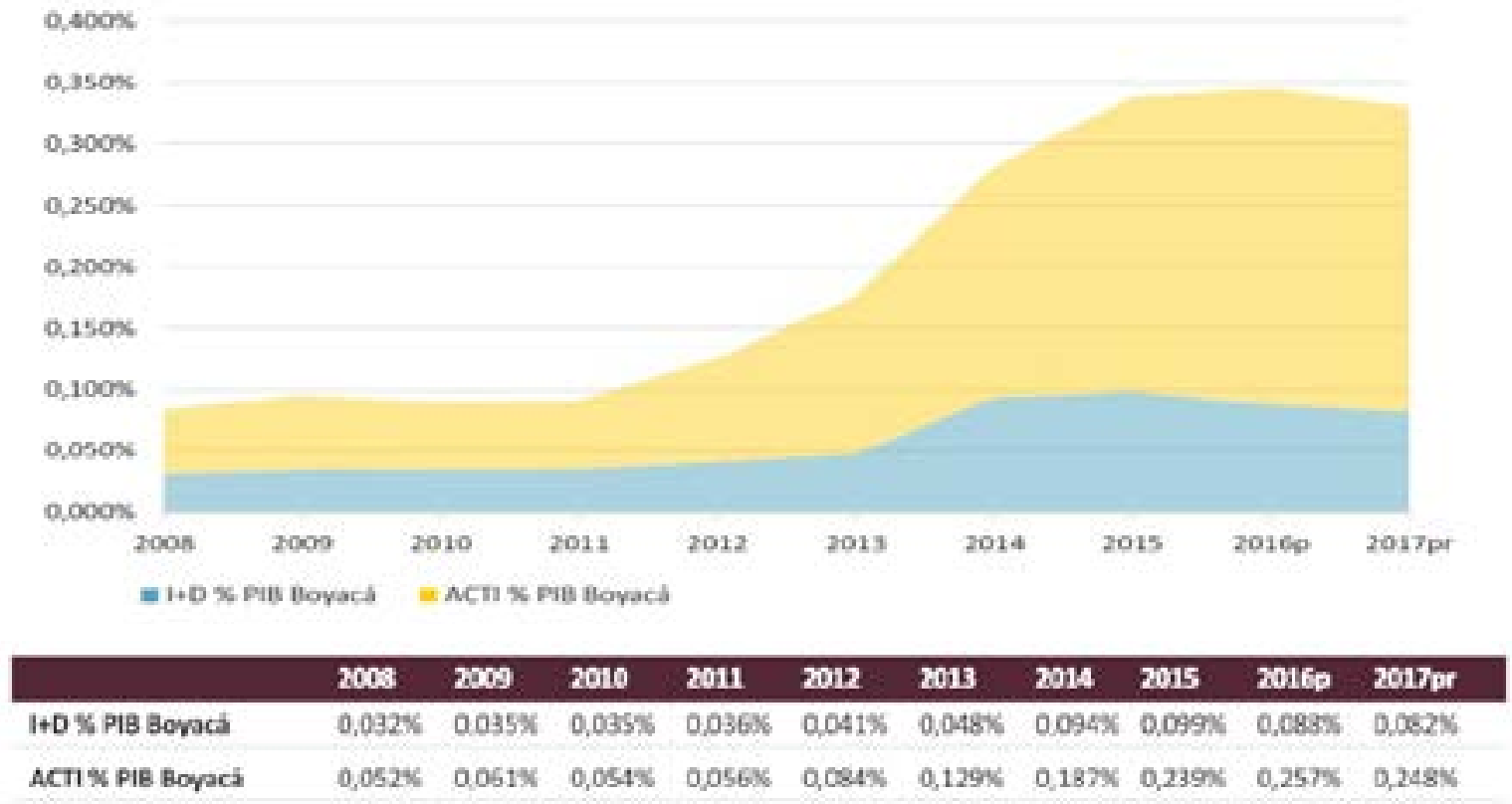

Fuente: OCITEB y UPTC, (2017). 
Por otra parte, de acuerdo con los datos del informe de "indicadores de ciencia, tecnología e innovación-ficha departamental-2017" de COLCIENCIAS en la Figura 11 se presentar la inversión en actividades de ciencia, tecnología e innovación para el Departamento con un monto de 93.340 millones en el 2017 (https:// bit.ly/2o5mF4f) que corresponde al $12,42 \%$ de la inversión de la región centro oriente y al 1,6\% del total nacional.

Figura 11. Inversión en Actividades de Ciencia, tecnología e Innovación para el Departamento

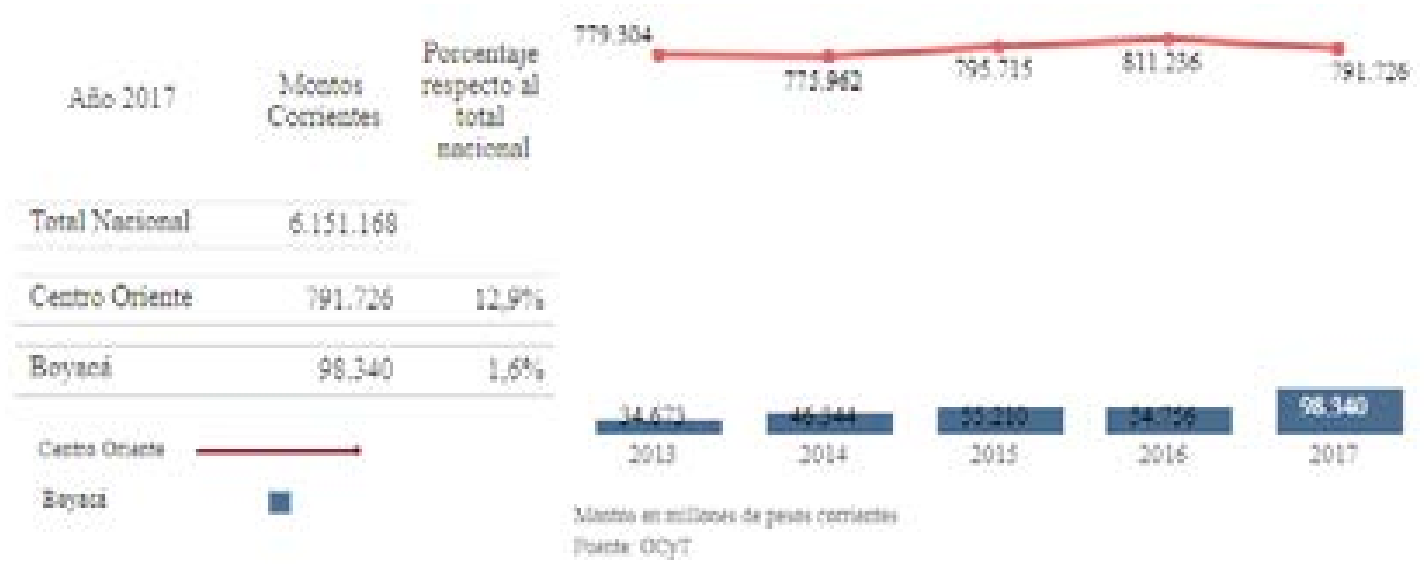

Fuente: OCyT (2017)

En la Figura 12 se presenta la inversión en Investigación, Desarrollo Tecnológico e Innovación para el departamento según el informe de la ciencia en cifras de COLCIENCIAS la inversión fue en el 2017 fue 62.573 millones (https://bit.ly/205mF4f) que corresponde al $17.75 \%$ de la inversión de la región centro oriente y al $1 \%$ del total nacional.

Figura 12. Inversión en Investigación,

Desarrollo Tecnológico e Innovación para el

Departamento

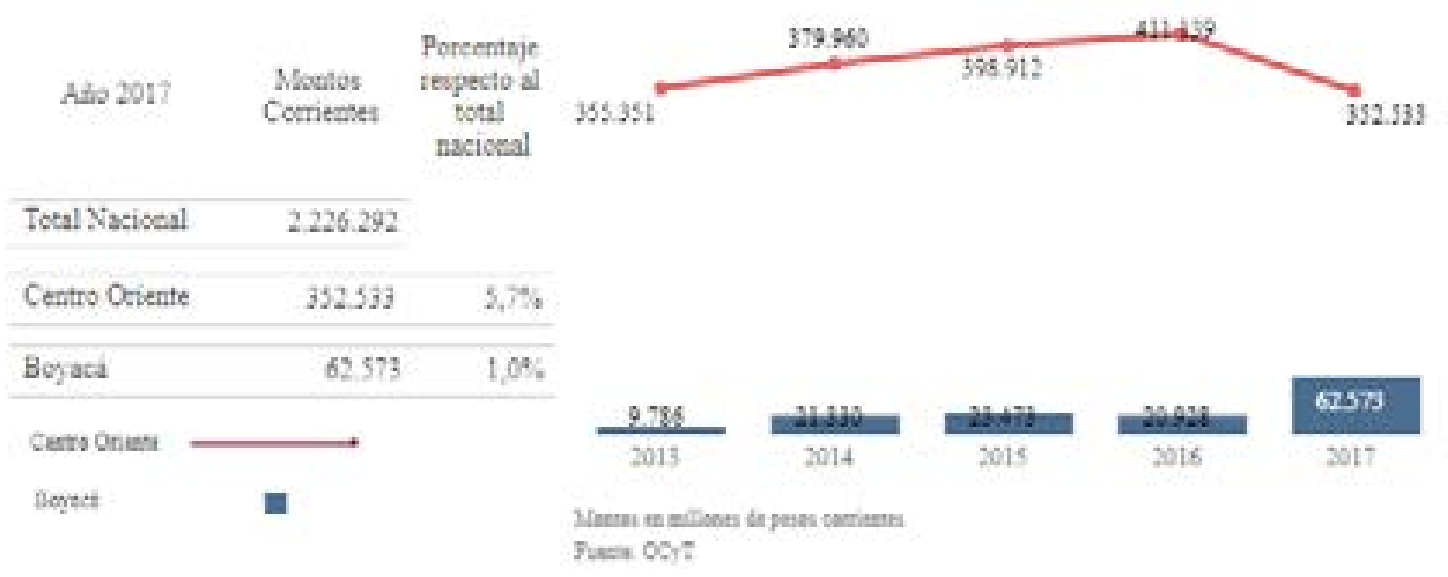

Fuente: OCyT (2017) 
En Boyacá al igual que en Colombia la capacidad en ciencia y tecnología medida a partir de los grupos de investigación activos, se encuentra centrada en las Instituciones de Educación Superior (IES) tanto públicas como privadas. De acuerdo con los resultados de la

Figura 13. Categorización de Grupos 2017 por categoría y área de conocimiento para el departamento de Boyacá convocatoria 781 de 2017 de COLCIENCIAS (Ver Figura 13), Boyacá reportó 173 grupos de investigación activos en 2017. Las cifras hacen evidente el bajo porcentaje de grupos de investigación del departamento con un $26.17 \%$ respecto a la región centro oriente y del 3.3\% respecto al total nacional.

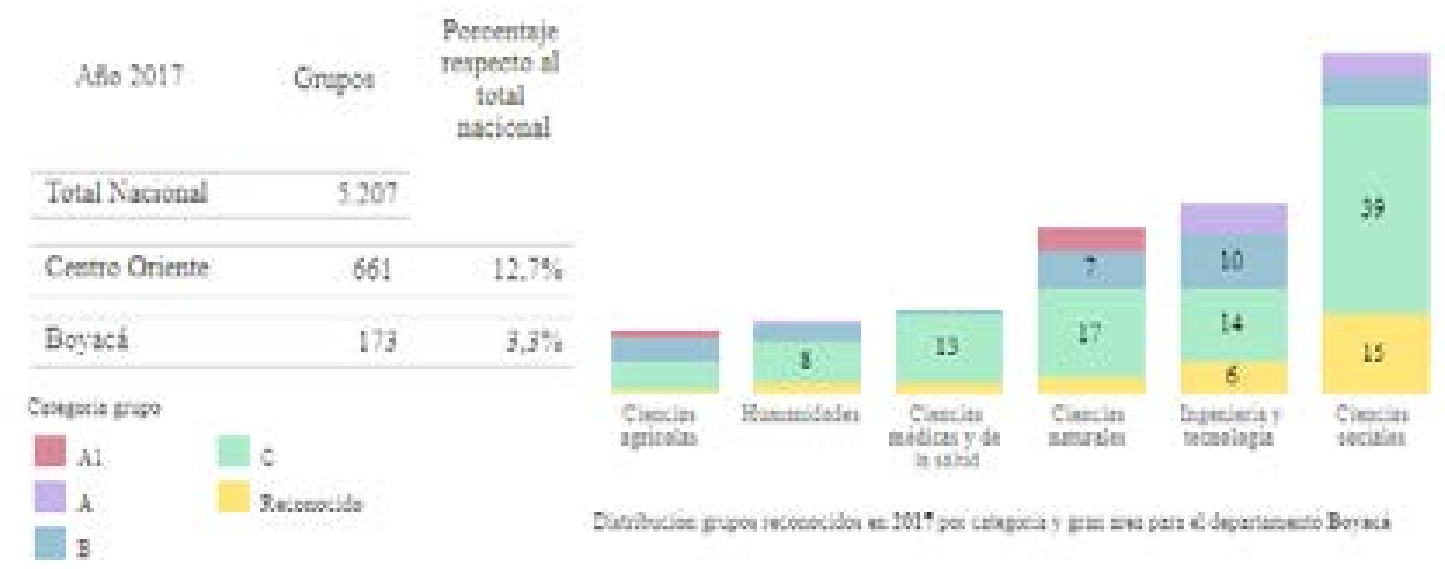

Fuente: COLCIENCIAS (2017)

Esta misma convocatoria muestra la categorización de investigadores por tipología y por área de conocimiento en donde se puede apreciar que el número de investigadores aún

Figura 14. Categorización de investigadores por tipología y área de conocimiento para el departamento de Boyacá

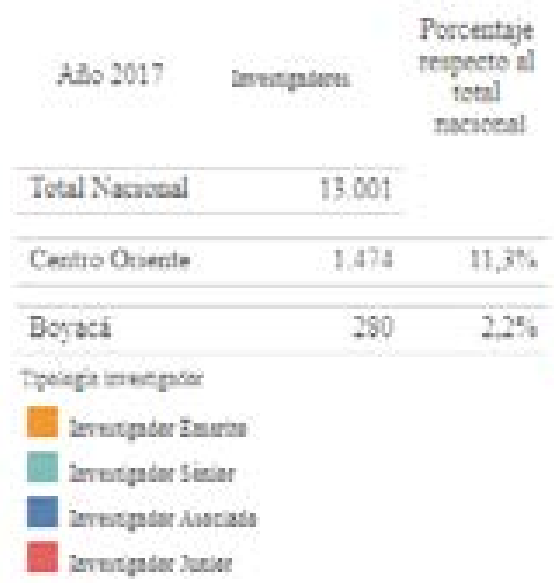

es muy bajo respecto a la región centro oriente con un $19 \%$ y muy inferior al total nacional con un $2.15 \%$ (ver Figura 14)

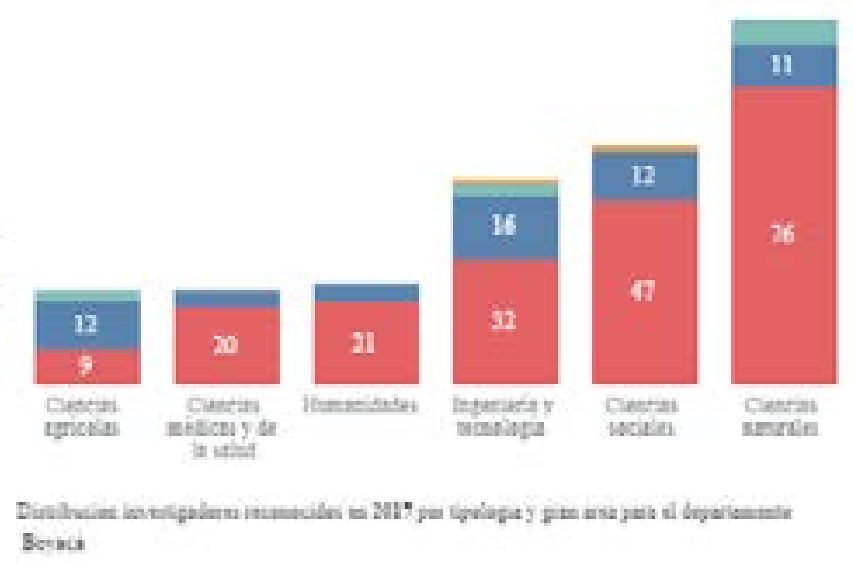

Fuente: COLCIENCIAS (2017) 
Por otra Parte, los resultados del informe de Indicadores de Ciencia, Tecnología e Innovación para Boyacá 2016-2017 (OCITEB \& UPTC, 2018), el promedio de investigadores por cada cien mil habitantes para el departamento fue de 12.6, es decir, aproximadamente 13 de cada cien mil habitantes, los cuales reportan estar vinculados a actividades de investigación. La participación del departamento en recurso humano relacionado con Investigación y Desarrollo (I+D), representado por los investigadores activos fue del $2.3 \%$ del total nacional, en número de doctores y en magister graduados en programas nacionales fue del $0.6 \%$ y $1.7 \%$ respectivamente.
Según el informe Instituto Colombiano para la Evaluación de la Educación (ICFES 2018), en las pruebas saber pro el departamento ocupó el tercer lugar con un puntaje de 9.62 de 10.0 puntos posibles; superado solamente por Bogotá que ocupó el primer lugar con 10.0 puntos y Caldas con 9.78 que ocupó el segundo lugar. Las competencias genéricas evaluadas se centraron en escritura, lectura crítica y razonamiento cuantitativo (https://bit. ly/2Oof4Z5)(Cordoba \& Garcia, 2018). En la Figura 15 se muestra el comportamiento del desempeño del departamento en cuanto al puntaje, lo cual se evidencian que desde el 2013 hasta el 2018 siempre ha estado entre los 5 primeros puestos a nivel nacional, exceptuando e 2014 que ocupó el 8 lugar.

Figura 15. Desempeño del Departamento en las pruebas Saber Pro 2013-2018

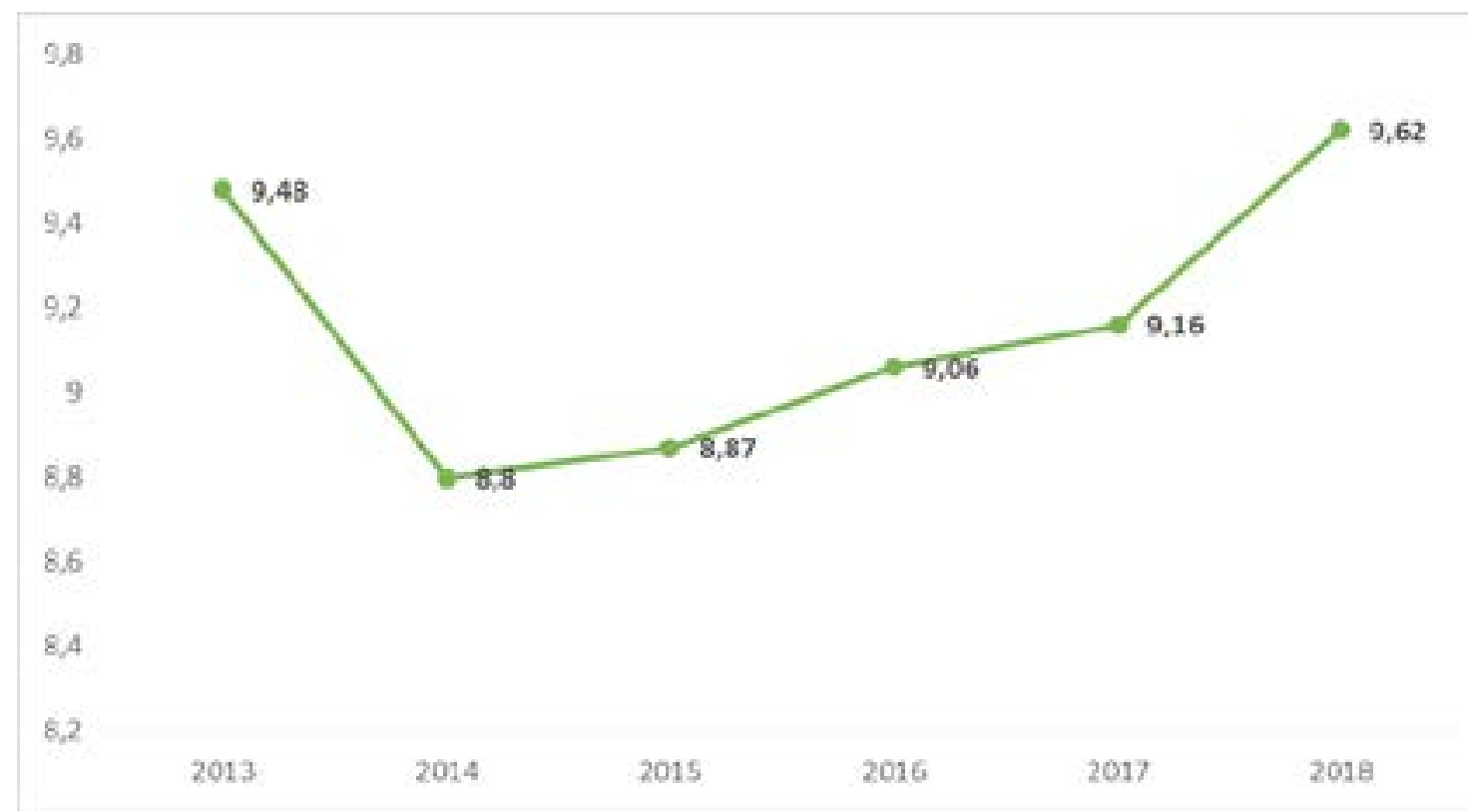

Fuente: ICFES (2018)

La Tabla 1 presenta el puesto ocupado en el departamento en las pruebas saber-pro desde el 2013 hasta el 2018. 
Tabla 1. Desempeño del Departamento en las pruebas Saber Pro 2013-2018

\begin{tabular}{ccccccc}
\hline Años & $\mathbf{2 0 1 3}$ & $\mathbf{2 0 1 4}$ & $\mathbf{2 0 1 5}$ & $\mathbf{2 0 1 6}$ & $\mathbf{2 0 1 7}$ & $\mathbf{2 0 1 8}$ \\
\hline Puesto & 2 & 8 & 3 & 4 & 5 & 3 \\
Puntaje & 9,48 & 8,8 & 8,87 & 9,06 & 9,16 & 9,62 \\
\hline
\end{tabular}

Fuente: ICFES (2018)

\section{PILAR 5. SOFISTICACIÓN DEL NEGOCIO}

El último pilar en el subíndice insumos, establece qué tan propensas son las empresas a realizar actividades innovadoras. Este pilar aborda elementos como el capital humano vinculado a sector productivo, relaciones de cooperación para innovar y la apropiación de conocimiento para el desarrollo de innovaciones (DNP et al., 2015). Para este se analiza la información del subpilar enlaces de innovación y específicamente el indicador colaboración en investigación entre U-E-E (\%) dado que está relacionados con las actividades de transferencia y relacionamiento del sector empresarial con las IES.

\section{SUBPILAR ENLACES DE INNOVACIÓN}

Este subpilar mide el nivel de colaboración entre empresas y organizaciones de conocimiento, la especialización industrial de las economías locales, el nivel de inversión en I+D financiado desde el extranjero y la proporción de empresas que cooperan con organizaciones internacionales (DNP et al., 2015). El análisis en este punto se centra en el indicador de colaboración en investigación entre U-E-E (\%).

A partir del año 2012 se implementa el Sistema General de Regalías (SGR) para la financiación de proyectos y programas de Ciencia, Tecnología e Innovación (CTel) a nivel nacional, recursos destinados por mandato constitucional y que estipulan el $(10 \%)$ de los recursos que el Estado Colombiano obtiene por la explotación de los recursos naturales no renovables. En este sentido, la apuesta del departamento en 2017 bajo la estrategia de alianzas y sensibilización para la innovación de COLCIENCIAS fueron apoyadas105 empresas en procesos de innovación en el departamento de Boyacá que corresponde al $29.9 \%$ de la región centro oriente y al $4.8 \%$ a nivel nacional (https://bit.ly/2o5mF4f). Por otra parte, la participación en la convocatoria de beneficios tributarios la participación del departamento fue del $0.3 \%$ de proyectos aprobados y del $0.1 \%$ por monto asignado a nivel nacional y del $2.1 \%$ de proyectos aprobados y del $0.4 \%$ por monto asignado de la región centro oriente. Finalmente el departamento generó convocatorias para apoyar proyectos de I+D+i en cooperación con COLCIENCIAS con el fin de fortalecer temas relevantes para la regios y para propiciar la articulación Universidad empresa (OCITEB \& UPTC, 2018)

\section{RETOS DE LAS IES DEL DEPARTAMENTO DE BOYACÁ FRENTE A LOS RESULTADOS DE INNOVACIÓN}

A pesar de la mejora del desempeño de los insumos de innovación que presenta los indicadores de índice departamental de innovación para el departamento de Boyacá, aún no se ha logrado impactar a los indicadores relacionados con los resultados de innovación, como se evidencia en la Figura 3 en la cual se observa que los indicadores relacionados con este subíndice están en el nivel medio y bajo de desempeño. Este subíndice se compone de dos 
pilares: producción de conocimiento y tecnología y producción creativa. Este estudio se enfoca en el subpilar de generación de conocimiento que corresponden al pilar de producción de conocimiento y tecnología.

\section{PILAR 6. PRODUCCIÓN DE CONOCIMIENTO Y TECNOLOGÍA}

La producción de conocimiento y tecnología se sustenta en tres subpilares: creación del conocimiento, impacto del conocimiento, y difusión del conocimiento. El subpilar generación de conocimiento se relaciona con las dimensiones que miden generación de conocimientos científicos y tecnológicos, el subpilar impacto del conocimiento incluye estadísticas que representan el efecto de las actividades de innovación a nivel micro y macro finalmente el subpilar difusión de conocimiento intenta medir ciertas características asociadas a sectores de alto contenido tecnológico (DNP et al., 2015). Teniendo en cuenta el alcance de trabajo el estudio se centra en el indicador de creación del conocimiento.

\section{SUBPILAR GENERACIÓN DE CONOCIMIENTO}

Este subpilar se encuentra relacionado con todas aquellas dimensiones utilizadas para medir la generación de conocimientos científicos y tecnológicos, entre ellas: solicitudes de patentes, solicitudes de modelos de utilidad, de artículos científicos y técnicos publicados en revistas indexadas, índice $\mathrm{H}$ medida para evaluar la calidad y el nivel de la producción científica y el porcentaje de empresas innovadoras en sentido amplio y estricto (DNP et al., 2015).

En la Figura 16 se observa la evolución entre el periodo comprendido del 2008 al 2018 de solicitud de patentes de invención en el departamento de Boyacá (residentes y no residentes) respecto a la solicitud a nivel nacional. Se puede observar la baja participación en este tipo de protección de propiedad industrial en el departamento, con un incremento en los últimos cinco años, pero aún muy bajo respecto al total nacional que representa el $0.14 \%$ de las patentes solicitadas en el país en el periodo analizado.

Figura 16. Solicitud de patentes de invención del 2008 al 2018

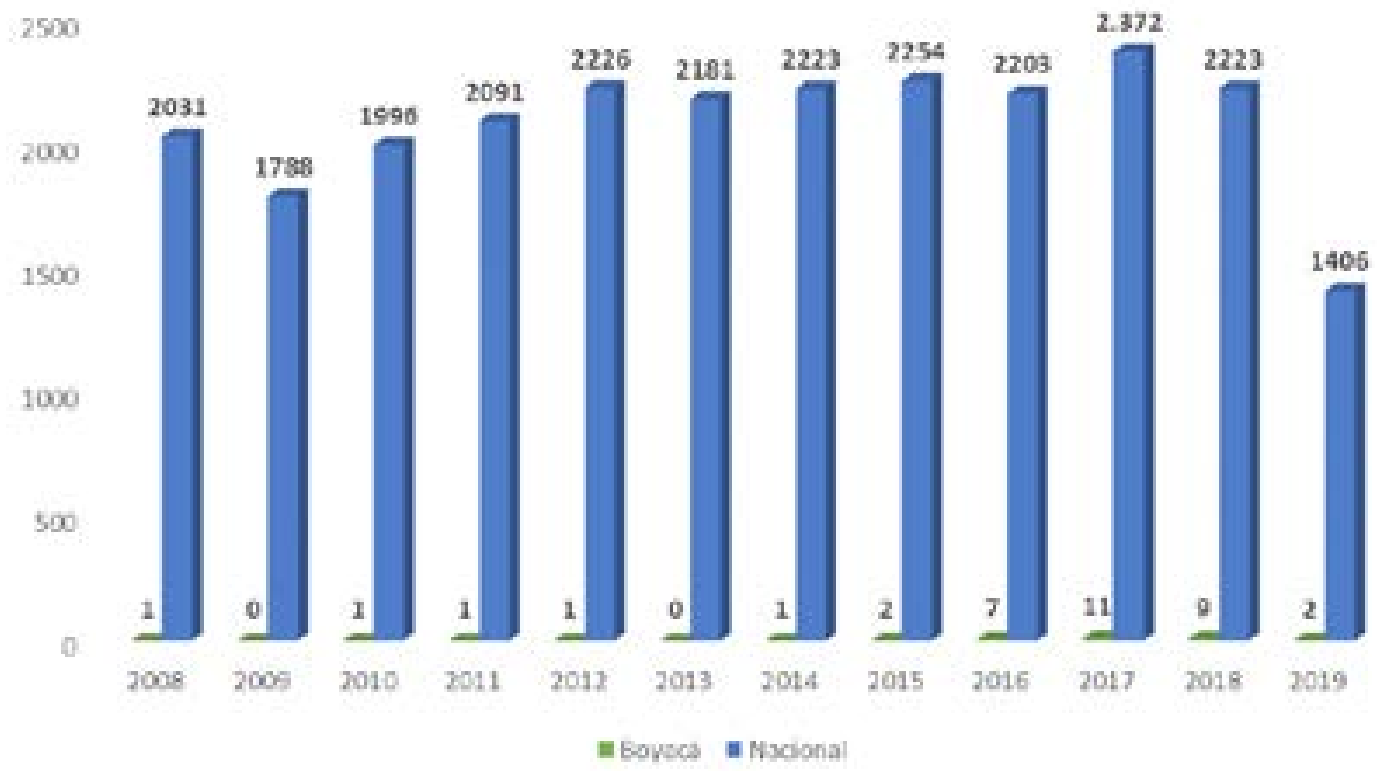

Fuente: Elaboración propia tomando información de la SIC (https://bit.ly/2Lv50cB) 
Las patentes de modelo de utilidad en el periodo comprendido del 2008 al 2018 para el departamento de Boyacá (residentes y no residentes) respecto a la solicitud a nivel nacional, también se evidencia en la Figura 17 la baja participación respecto al total nacional con un $0.9 \%$

Figura 17. Solicitud de patentes de modelo de utilidad del 2008 al 2018

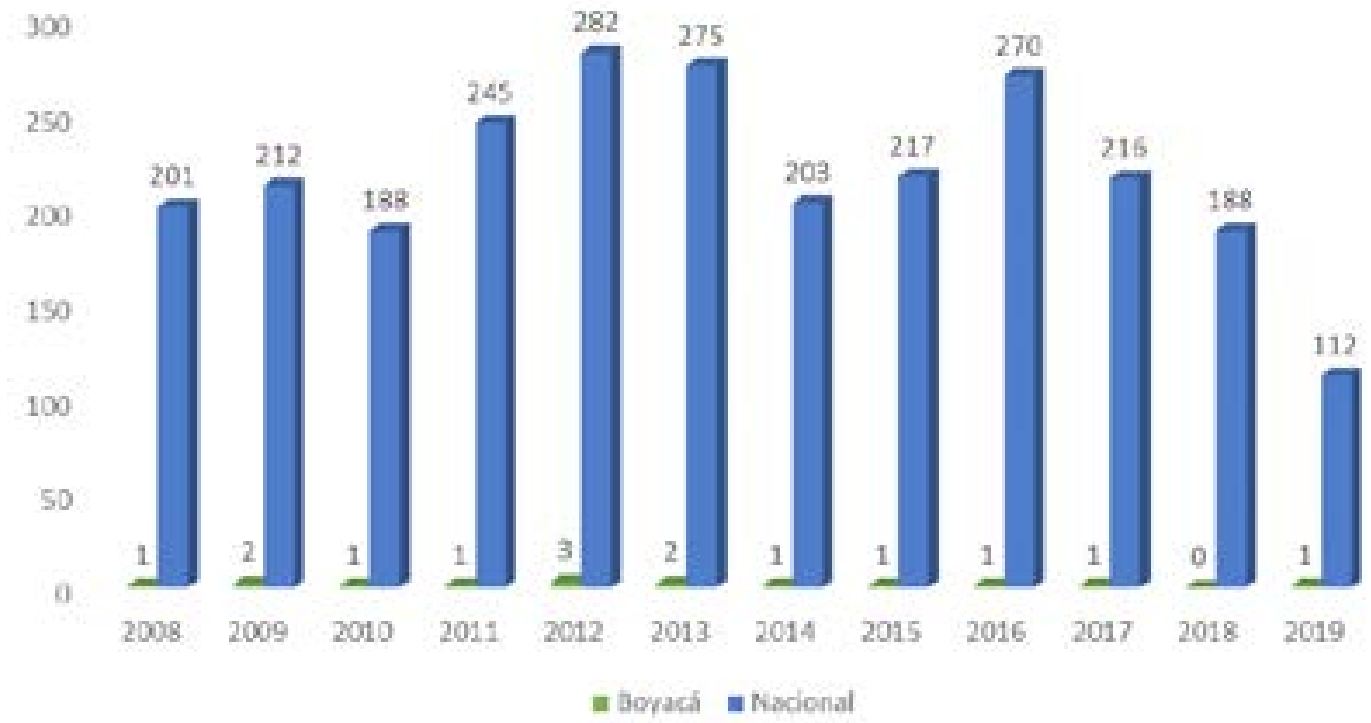

Fuente: Elaboración propia tomando

información de la SIC (https://bit.ly/2Lv50cB)

El número de documentos publicados, dan cuenta la generación de conocimientos a través de documentos en revistas de alta visibilidad internacional y que por lo tanto se encuentran indexadas en las principales bases de datos de revistas científicas (Web of Science Core Collection, SciELO y Scopus). Este es uno de los indicadores de resultado de las actividades de I+D más utilizados, el cual permite conocer las dinámicas en generación de conocimientos científicos en el país o región. Una mirada más profunda de estos documentos permite observar tenencias predominantes, las instituciones más productivas, y el impacto sobre la comunidad científica. Como se presenta en la Figura 18, respecto a las publicaciones de artículos en bases de datos académicas de alto impacto como Web of Science, SciEL y Scopus a nivel departamental presenta un aumento constante y a pesar de que la proporción respecto al total nacional es baja, es necesario tener en cuenta la proporción de IES en el departamento y el número de investigadores son factores relevantes para este resultado. 
Figura 18. Artículos publicados en bases de datos internacionales del 2007 al 2017

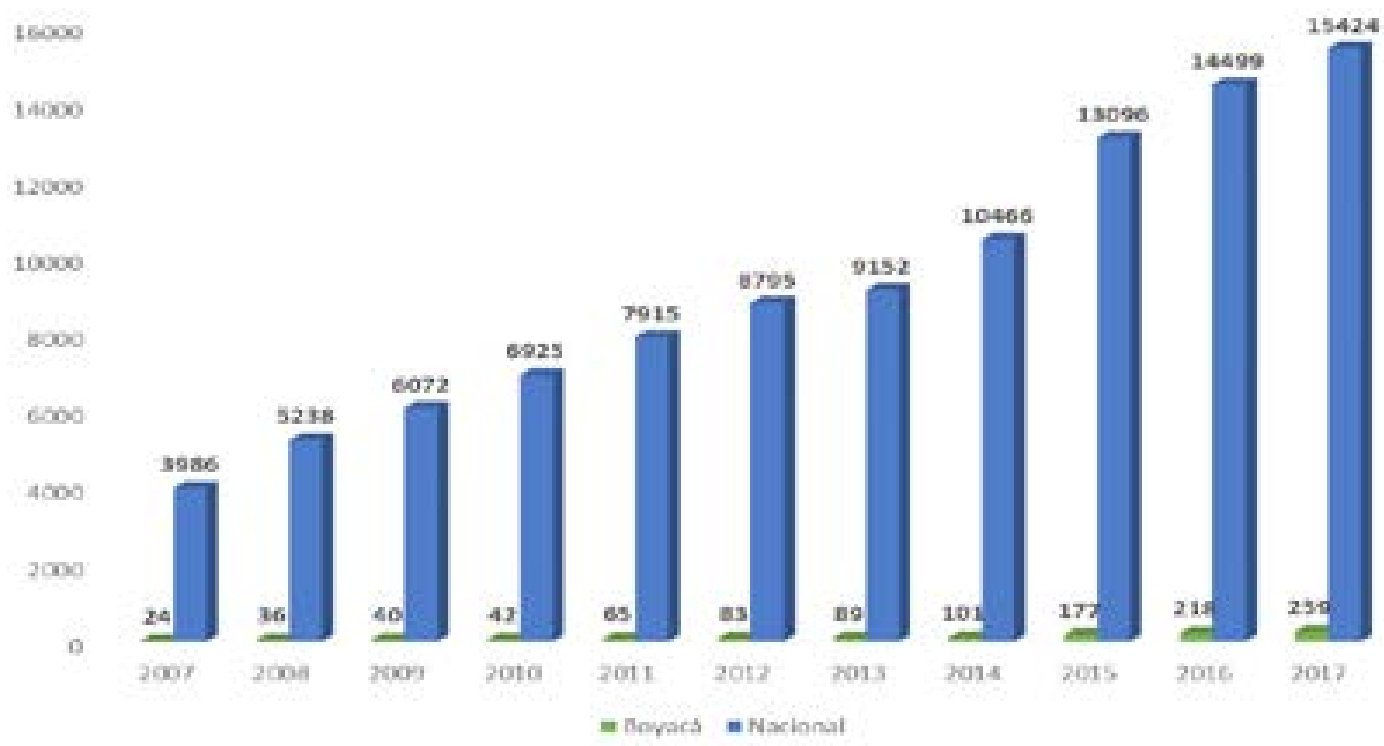

Fuente: Elaboración propia tomando información del portal de datos del observatorio de ciencia y tecnología (https://bit.ly/2MhELrt)

El índice $\mathrm{H}$, es el indicador que refleja el promedio de veces que los documentos han sido citados por los otros documentos dentro de la misma base de datos, desde el inicio de su publicación. Para los documentos más antiguos, al estar disponibles por un lapso de tiempo mayor, aumentan las posibilidades de ser citados, pero también se incrementa el riesgo de obsolescencia. En la Figura 19 se muestra la evolución de las citaciones de los documentos de los investigadores con filiación en el departamento respecto a las nacionales mostrando un desempeño muy favorable.

Figura 19. Promedio de citas totales de documentos publicados del 2007 al 2017

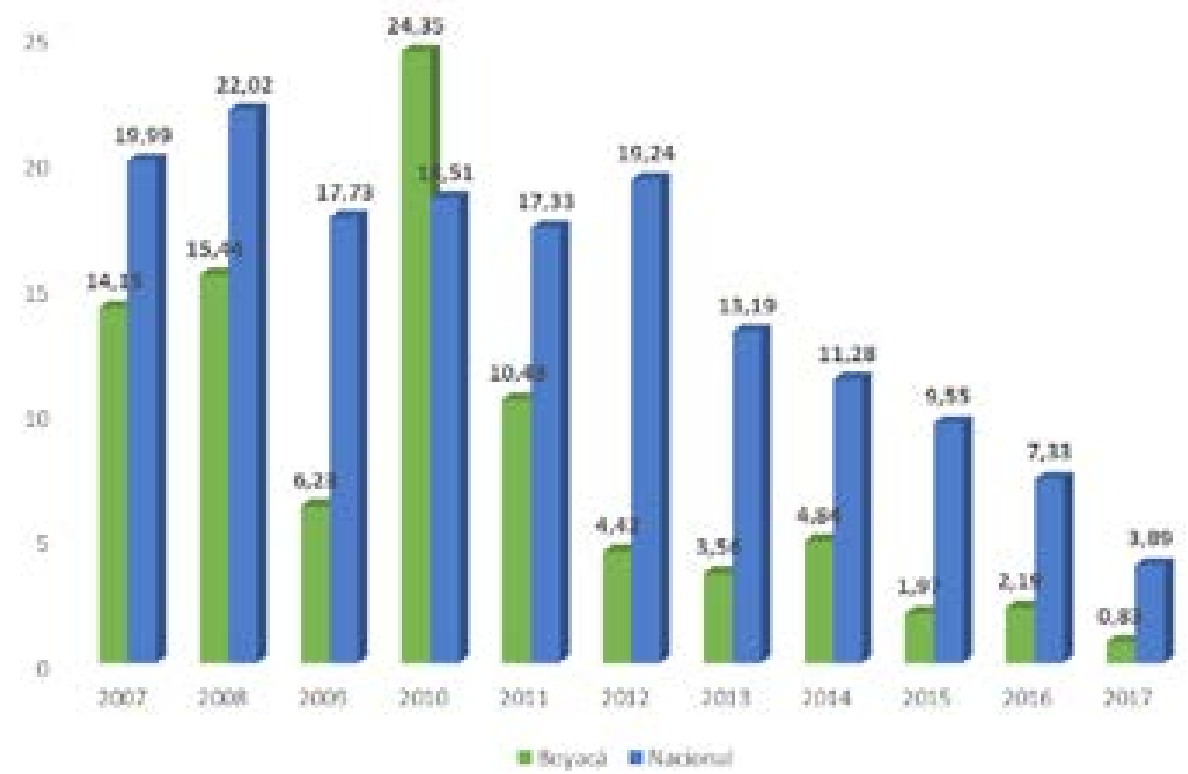

Fuente: Elaboración propia tomando información del portal de datos del observatorio de ciencia y tecnología (https://bit.ly/2MhELrt) 


\section{CONCLUSIONES Y RECOMENDACIONES}

- El análisis que se desarrolla en este trabajo tiene dos propósitos. En primer lugar, se busca profundizar en las relaciones generales que pueden establecerse entre las funcionales misionales de las IES y las capacidades de innovación medidas por el IDIC para el departamento de Boyacá y en segundo lugar, profundizar en el análisis de los resultados de innovación alcanzados por el Departamento respecto a los insumos de innovación en marcados en los indicadores relacionados con la unidad de análisis de este trabajo.

- Los resultados del informe del IDIC 2018 revelan que el departamento de Boyacá tiene desempeños altos en el subíndice de insumos, pero muy bajos en el subíndice de resultados; generando una razón de eficiencia de 0.42 , por lo tanto, se considera una combinación poco eficiente para la generación de innovación teniendo en cuenta la calidad y cantidad de los insumos empleados para obtener resultados robustos. Sin embargo, el comportamiento esta razón de eficiencia para el departamento se puede justificar por la misma dinámica de las actividades de $1+D+i$, por cuanto la apuesta del departamento para mejorar las capacidades e insumos se ha incentivado en los últimos años, por lo tanto, es de esperarse que el impacto del subíndice de resultados se empiece evidenciar.

- Es importante resaltar que a pesar de que Boyacá ha permanecido en el puesto 12 del IDIC desde el 2015 al 2018, permanecer en este rango ha sido un reto importante por cuanto en 2015 el estudio contemplo 25 departamentos mientras que en 2018 se incluyen 31 departamentos en el análisis $y$ adicionalmente los indicadores se han venido refinando y mejorando para facilidad de la medición.

- De los indicadores analizados en este trabajo los catalogados en el IDIC-2018 con alto desempeño se destacan: 1. la tasa de cobertura bruta en educación superior, evidenciada en la tendencia creciente de las matriculas en educación superior en el departamento en el periodo comprendido entre el 2010 al 2017, mostrando la apuesta del departamento para el fortalecimiento de educación superior. 2. Artículos en publicaciones científicas y técnicas presenta un aumento constante y a pesar de que la proporción respecto al total nacional es baja, es necesario tener en cuenta la proporción de IES en el departamento y el número de investigadores son factores relevantes para este resultado.

- Por otra parte, los indicadores que se presentan mayores debilidades con un desempeño medio-bajo son: gasto en investigación y desarrollo como porcentaje del PIB, desempeño en pruebas Saber de educación terciaria, colaboración en investigación entre empresas y otras organizaciones de conocimiento (\%) y solicitudes de modelos de utilidad por millón de habitantes. Es importante resalta que de los indicadores seleccionados para este trabajo no se reporta ninguno con desempeño bajo (DNP \& OCyT, 2018)

- En general, se deben aprovechar las oportunidades que potencien el desarrollo del departamento a través el diseño de estrategias orientadas a cumplir los programas en mención, en los que la administración municipal y la academia propendan por espacios que integren a los sectores industriales del departamento. Por consiguiente, se resalta la importancia de fomentar la innovación desde una perspectiva estratégica para lograr una economía dinámica y competitiva que disminuya la brecha que existe en materia de innovación a nivel nacional e internacional. 


\section{REFERENCIAS}

Castillo, H. (2010). El modelo de la triple hélice como un medio para la vinculación entre la universidad y empresa. Revista Nacional de administración, 1(1), 85-94.

Cordoba, R., \& Garcia, H. (2018). Índice departamental de Competitividad. Consejo privado de competitividad. Retrieved from https://compite.com.co/wp-content/ uploads/2019/03/LIBRO-CPC_IDC_2018_ WEB.pdf

DNP, D. N. de P. (2018). Índice Global de Innovación 2018, 23.

DNP, D. N. de P., \& OCyT, O. de C. у T. (2018). Indice de Innovación para Colombia (IDIC), 2018. Retrieved from www.ocyt.org.co

DNP, OCyT, \& Consultores, C. (2015). Índice Departamental de Innovación para Colombia (IDIC), 2015, (Idic), 152. Retrieved from https://colaboracion. dnp.gov.co/CDT/Prensa/Publicaciones/ Índice de Innovación Departamental para Colombia.pdf

Dutta, S., Lanvin, B., \& Wunsch-Vincent, S. (2018). Global Innovation Index 2018. Retrieved from http://creativecommons. org/licenses/by-nc-nd/3.0/igo/.

Etzkowitz, H., \& Leydesdorff, L. (2000). The dynamics of innovation: from National Systems and "Mode 2" to a Triple Helix of university-industry-government relations. Research Policy, 29(2), 109-123. https:// doi.org/10.1016/S0048-7333(99)00055-4

Gunasekara, C. (2006). Reframing the Role of Universities in the Development of Regional Innovation Systems. The Journal of Technology Transfer, 31(1), 101-113. https://doi.org/10.1007/s10961-005-50164
OCITEB, \& UPTC. (2018). Boyaca en Cifras (Vol. 112). https://doi.org/10.1192/ bjp.112.483.211-a 